\section{„Idiopathische“ Lungenerkrankungen haben häufig Ursachen in der Umwelt}

\author{
Die Diagnose „idiopathische interstitielle Pneumonie/Fibrose“ \\ sollte nicht vorschnell erfolgen. Gar nicht so selten steckt nämlich \\ eine allergisch bedingte Überempfindlichkeit gegen bestimmte \\ (häusliche) Umweltfaktoren dahinter.
}

$\mathrm{U}$ nter der Bezeichnung idiopathische interstitielle Pneumonie/Fibrose (IIP/F) werden eine Reihe von Lungenerkrankungen subsumiert, die sich in Morphologie, Schweregrad und Behandelbarkeit stark unterscheiden. Über 16 Jahre gingen amerikanische Autoren möglichen allergischen Faktoren als Ursache einer IIP/F von insgesamt 74 Patienten nach.

Neben den gängigen klinischen Untersuchungen wurden die Patienten nach spezifischen Umweltbedingungen vor allem im häuslichen Umfeld gefragt - insbesondere, ob im zeitlichen $\mathrm{Zu}$ sammenhang mit dem Ausbruch der Symptomatik dort eine Veränderung stattgefunden hatte. Auch wurden das

\section{Allgemeine antimikrobielle}

Prophylaxemethoden

Betroffenen Personen wurden folgende Maßnahmen zur häuslichen Lufthygiene empfohlen:

_ Tägliche Lüftung der Wohnung

- 40-50\% Luftfeuchtigkeit in der Wohnung

— Meiden von Haustieren und Pflanzen

— Feuchtigkeit produzierende Faktoren vermeiden (z. B. Aquarien, Zimmerspringbrunnen)

— Wasserabflüsse und Abfalleimer wöchentlich mit antimikrobiellem Reiniger behandeln

— "Chaos" in der Wohnung vermeiden

— Müll täglich aus der Wohnung bringen

— Hochwirksame Filter benutzen und regelmäßig reinigen

— Auffangbehälter für Kondensierflüssigkeit von Kühlschränken und Klimaanlagen monatlich prüfen und reinigen

— Jeden Monat den Raum um mögliche Feuchtigkeitsquellen, z. B. an Abflüssen, hinter dem Wäschetrockner etc., prüfen und reinigen

Nach RL Jacobs et al. häusliche Umfeld bzw. der Arbeitsplatz inspiziert, in einigen Fällen zusätzlich serologische Tests durchgeführt. Anschließend sollten mögliche Allergenquellen der Umgebung eliminiert werden oder die Patienten umziehen.

Bei 69 der 74 Patienten konnte eine organische Kontamination des häuslichen Umfelds als Ursache für die Erkrankung festgestellt werden, bei drei waren es Faktoren am Arbeitsplatz. Allergische Reaktionen waren meist nur mit speziell aus der Umgebung der Patienten hergestellten Allergenextrakten nachzuweisen. Nach Beseitigung der schädlichen Einflüsse waren 50 Patien-
A nfang der 90er Jahre wurden im Rahmen des European Community Respiratory Health Survey (ECRHS) ein Teil der erwachsenen Teilnehmer ( $\mathrm{n}=7.882$ ) strukturiert u. a. zu Passivrauchen und Lungensymptomen befragt. Daneben wurden diese Patienten einer Spirometrie und einem Methacholin-Provokationstest unterzogen und Gesamt- und spezifisches IgE bestimmt.

Die Prävalenz von Passivrauchen am Arbeitsplatz nahm von Norden (Schweden 2,5\%) nach Süden (Spanien 53,8\%) zu. Das Passivrauchen war signifikant assoziiert mit Symptomen wie nächtlicher Brustenge (OR 1,28) und nächtlicher Atemlosigkeit (OR 1,30) sowie Atemlosigkeit nach körperlicher ten ohne weitere medikamentöse Behandlung beschwerdefrei. Acht weitere Patienten benötigten weiterhin Steroide, sechs von ihnen waren allerdings in der belastenden Umgebung geblieben und hatten keine Sanierungsmaßnahmen durchgeführt. Insgesamt 16 Patienten verstarben - aus unterschiedlichen Gründen - in der 16-jährigen Beobachtungszeit.

Vor allem eine zu große Feuchtigkeit in den Wohnungen und damit mikrobieller Befall von Tapeten, Teppichen und Einrichtungsgegenständen war mit dem Auftreten der Lungenerkrankungen assoziiert - undichte Dächer bei alten sowie Baufeuchte in neuen Häusern trugen dazu bei. Auch mikrobiell verunreinigte Klimaanlagen konnten als Ursache identifiziert werden.

Jacobs RL et al.: Organic antigen-induced interstitial lung disease: diagnosis and management. Ann Allergy Asthma Immunol 2002; 88: 30-41

\title{
Bronchiale Hyperreagibilität durch Passivrauchen am Arbeitsplatz
}

\section{Der schädliche Einfluss von Passivrauchen auf die Lungenfunktion ist bei Kindern ausreichend belegt. Ein ähnlicher Zusammenhang ergibt sich auch bei Erwachsenen - zumindest für Passivrauchen am Arbeitsplatz.}

Beanspruchung (OR 1,25). Auch Asthma und eine bronchiale Hyperreaktivität waren mit Passivrauchen korreliert. Kein signifikanter Zusammenhang ergab sich beim IgE. Auch war kein Zusammenhang mit Passivrauchen zu Hause erkennbar.

\section{Fazit}

Ein Rauchverbot am Arbeitsplatz könnte zur Lungengesundheit beitragen. $b k$

Janson C et al.: Effect of passive smoking on respiratory symptoms, bronchial responsiveness, lung function, and total IgE in the European Community Respiratory Health Survey: a cross-sectional study. Lancet 2001: 358: 2103-9 